\title{
Regular Sparse Array Direction of Arrival Estimation in One Dimension
}

\author{
Ferre Knaepkens, Annie Cuyt, Wen-shin Lee, Member, IEEE, and Dirk I.L. de Villiers, Senior Member, IEEE
}

\begin{abstract}
Traditionally regularly spaced antenna arrays follow the spatial Nyquist criterion to guarantee an unambiguous analysis. We present a novel technique that makes use of two sparse non-Nyquist regularly spaced antenna arrays, where one of the arrays is just a shifted version of the other. The method offers several advantages over the use of traditional dense Nyquistspaced arrays, while maintaining a comparable algorithmic complexity for the analysis. Among the advantages we mention: an improved resolution for the same number of receivers and reduced mutual coupling effects between the receivers, both due to the increased separation between the antennas. Because of a shared structured linear system of equations between the two arrays, as a consequence of the shift between the two, the analysis of both is automatically paired, thereby avoiding a computationally expensive matching step as is required in the use of so-called co-prime arrays.

In addition, an easy validation step allows to automatically detect the precise number of incoming signals, which is usually considered a difficult issue. At the same time, the validation step improves the accuracy of the retrieved results and eliminates unreliable results in the case of noisy data. The performance of the proposed method is illustrated with respect to the influence of noise as well to the effect of mutual coupling.
\end{abstract}

Index Terms-Array Antennas, Direction of Arrival Estimation, Sparse Arrays.

\section{INTRODUCTION}

D IRECTION of arrival (DOA) estimation, using array antenna systems, is a topic of increasing interest in a variety of applications including radar, remote sensing, radio frequency interference mitigation, and smart wireless networks [1]-[3]. One of the most well-known limitations in regularly spaced antenna array systems is, arguably, the requirement that the elements should have spacing closer than a half-wavelength (the spatial Nyquist criterion) in order to avoid aliasing resulting in ambiguous arrival angle estimates. Unique results can be obtained for larger spacings if a limited range of near-broadside receiving angles are considered, or if the antenna element patterns exhibit zeros in the end-fire directions, but in general this still limits the allowable spacing to distances close to the Nyquist limit.

Ferre Knaepkens and Annie Cuyt are with the Department of Mathematics and Computer Science, University of Antwerp (CMI), Antwerp, Belgium. email: \{ferre.knaepkens, annie.cuyt $\} @$ uantwerpen.be.

Wen-shin Lee is with the Division of Computing Science and Mathematics, University of Stirling, Stirling, United Kingdom, and the Department of Mathematics and Computer Science, University of Antwerp (CMI), Antwerp, Belgium. e-mail: wen-shin.lee@stir.ac.uk

Dirk de Villiers is with the Department of Electrical and Electronic Engineering, Stellenbosch University, Stellenbosch, South Africa. e-mail: ddv@sun.ac.za

Manuscript received XXXX XX, 2018; revised XXXX XX, 2018. (c) 2020 IEEE. Personal use of this material is permitted. Permission from IEEE must be obtained for all other uses, in any current or future media, including reprinting/

republishing this material for advertising or promotional purposes, creating new collective works, for resale or redistribution to servers or lists, or reuse of any copyrighted component of this work in other works.

A wealth of research is available in the antenna array and signal processing literature on algorithms which reliably estimate the DOA of incoming signals in real world noisy environments. The most popular ones are the MUltiple SIgnal Classification (MUSIC) [4], the EStimation Parameter via Rotational Invariance Technique (ESPRIT) [5] and the Matrix Pencil method [6].

The requirement of close antenna element spacing limits the achievable resolution of an array system with a fixed number of receivers (or sensors) according to the well-known diffraction (or Rayleigh) limit

$$
\Delta_{\phi} \approx \frac{\lambda}{D}
$$

where $\Delta_{\phi}$ is the resolution in radians, $\lambda$ is the operating wavelength, and $D$ is the length of the array (in the linear array case). A system with $M$ elements spaced at $\lambda / 2$ will thus have a resolution limited to $\Delta_{\phi} \approx 2 /(M-1)$, explicitly stating the inverse relationship between resolution and the number of elements. Several sparse array configurations, with elements spaced at distances larger than the Nyquist limit, have been proposed including minimum redundancy arrays (MRAs) [7], nested arrays [8], and co-prime arrays [9]. All these configurations have the advantage of increased resolution, for a given number of sensors, over that of an ULA. The main disadvantage, however, is the increased computational burden associated with estimating the correct angles of arrival in noisy systems, which follows from a combinatorial matching problem that arises when resolving the introduced aliasing.

A disadvantage of densely spaced antenna elements is the effect of mutual coupling, which causes uncertainty in the so-called array manifold, that is the collection of received steering vectors from all possible directions. This in turn leads to errors in the estimated arrival angles, which is normally reduced through extensive calibration of the system. Since accurate estimation of the installed mutual coupling matrix is difficult, in addition to often being time variant, many popular calibration techniques exist [10]-[13]. Typically, the computational cost of the mutual coupling estimation can be high, especially for iterative techniques. To overcome this, some methods that use auxiliary sensors have been proposed, but these come at a cost of reduced effective aperture [14], [15]. A comparison of the performance of several sparse-array methods in the presence of mutual coupling is given in [16].

In this paper we present a DOA estimation method that exploits some recent progress in exponential analysis and completely removes the dense Nyquist spacing requirement in ULAs [17]. Removing the Nyquist requirement allows for 
larger spacing between individual antenna elements, leading naturally to improved angular resolution as well as generally reduced mutual coupling. The larger antenna element spacing implies an increase of the size of the antenna array, which depending on the application, may not pose a problem [9], [18], [19]. The method makes use of two interleaved sparse ULAs, where one of them is just a shifted version of the other. The set of angles and their aliases obtained from the first sparse ULA via exponential analysis, is subsequently intersected with information received from the second ULA via the solution of a structured linear system, to retrieve the correct directions. Due to introducing a second ULA that is a shifted version of the first one, a combinatorial search step that other coprime array methods require, is avoided. Instead the results of the two ULAs are directly linked by their computation. In addition to handling the aliasing problem of sparse arrays in an efficient manner, the proposed technique can automatically determine the number of received signals with no prior user input, and allows detection of fully correlated signals from different directions without any special treatment.

Note that any one-dimensional exponential analysis solver could be used on the first ULA. Examples are MUSIC [4], ESPRIT [5], the matrix pencil method [6], simultaneous QR factorization [20], a generalized overdetermined eigenvalue solver [21] and the approximate Prony method [22]-[24].

The paper is structured as follows. First in Section II the notation is introduced and the problem statement of DOA estimation is described. Subsequently, in Section III standard Nyquist-based exponential analysis is discussed and we introduce an approach to determine the number of received signals, called the sparsity in exponential analysis, via density based cluster analysis. Section IV considers sparse ULAs, for which a sub-Nyquist sampling technique is formulated. Finally, we conclude with some examples in Section V.

\section{PROBLEM Formulation}

Consider a ULA with $M$ antenna elements receiving $n$ $(2 n \leq M)$ narrow-band signals at frequency $\omega$, as illustrated for the $i$ th signal in Fig. 1. The antenna elements are omnidirectional and arranged equidistantly along the $x$-axis with a spacing $d<\lambda / 2$ to prevent aliasing. The objective is to determine the directions of the received narrow-band signals, in other words the angles $\phi_{i}$, as seen in Fig. 1. The narrowband

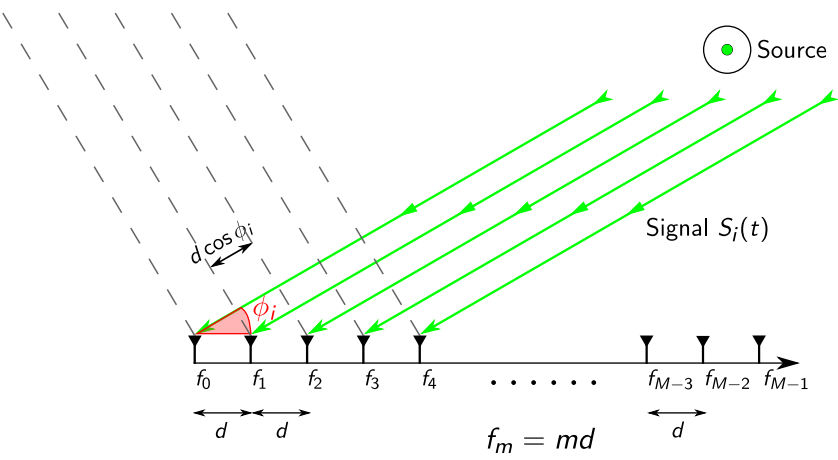

Fig. 1. Uniform linear array set-up for one incoming signal. signal $S_{i}(t)$ at time $t$ can be expressed as

$$
S_{i}(t)=s_{i}(t) \exp (j \omega t), \quad s_{i}(t)=a_{i}(t) \exp \left(j p_{i}(t)\right),
$$

where $a_{i}(t)$ and $p_{i}(t)$ denote the slowly varying amplitude and phase respectively. Assuming a plane wave incidence of $S_{i}(t)$ on the ULA, the time delay between consecutive antennas is given by $\tau_{i}=d \cos \left(\phi_{i}\right) / c$, where $c$ is the propagation velocity of the signal (speed of light in this case). We denote the output of the ULA at the $m$ th antenna element at time $t$ by $f_{m}(t)$, where

$$
f_{m}(t)=\sum_{i=1}^{n} S_{i}\left(t-m \tau_{i}\right), \quad \tau_{i}=\frac{d \cos \left(\phi_{i}\right)}{c}
$$

because of the time delay with respect to the reference antenna $f_{0}(t)$. The element $f_{0}$ receives the sum of the signals $S_{i}(t)$ at time $t$. The narrow-band assumption (the signal response does not change appreciably during the transit of the array) allows us to write

$$
s_{i}\left(t-m \tau_{i}\right) \approx s_{i}(t)
$$

or equivalently,

$$
\begin{aligned}
S_{i}\left(t-m \tau_{i}\right) & =s_{i}\left(t-m \tau_{i}\right) \exp \left(j \omega\left(t-m \tau_{i}\right)\right) \\
& \approx S_{i}(t) \exp \left(-j \omega m \tau_{i}\right),
\end{aligned}
$$

hence, the output $f_{m}(t)$ of the ULA, given by (2), can be rewritten as

$$
f_{m}(t)=\sum_{i=1}^{n} S_{i}\left(t-m \tau_{i}\right) \approx \sum_{i=1}^{n} S_{i}(t) \exp \left(-j \omega m \tau_{i}\right)
$$

Consequently, for every signal the time delay is expressed as a phase shift, depending on the direction $\phi_{i}$. From these time delays, which are identified by exponential analysis, it is possible to infer the directions $\phi_{i}$ of the received signals.

\section{STANDARD EXPONENTIAL ANALYSIS}

At a fixed time $t$ the output of the ULA is called a snapshot. This snapshot consists of the samples

$$
f_{m}(t)=\sum_{i=1}^{n} S_{i}(t) \exp \left(-\frac{j \omega m d \cos \phi_{i}}{c}\right),
$$

for $m=0, \ldots, M-1, M \geq 2 n$, and is used to formulate a one-dimensional exponential analysis problem. We introduce the notations

$$
\begin{aligned}
f_{m} & =f_{m}(t), & \alpha_{i} & =S_{i}(t), \\
\psi_{i} & =-\frac{j \omega \cos \phi_{i}}{c}, & \Psi_{i} & =\exp \left(\psi_{i} d\right) .
\end{aligned}
$$

We refer to the $f_{m}$ as the samples, the $\alpha_{i}$ as the coefficients, the $\psi_{i}$ as the exponents and the $\Psi_{i}$ as the base terms of the exponential analysis problem. Note that none of the notations depend on $t$ since, for the moment, we consider a single snapshot (hence $t$ is fixed). Now it is possible to rewrite (5) as

$$
f_{m}=\sum_{i=1}^{n} \alpha_{i} \Psi_{i}^{m}, \quad m=0, \ldots, M-1
$$


which can be solved for the $\alpha_{i}$ and $\psi_{i}$ under the assumption that the $\Psi_{i}$ are mutually distinct. It was shown in 1795 already by de Prony, that it is possible to solve the exponential analysis problem (7) from $2 n$ samples if the sparsity $n$ is given [25]. In its modern version, the method first recovers the values of the $\Psi_{i}$ as generalized eigenvalues, and subsequently solves a Vandermonde structured linear system to retrieve the coefficients $\alpha_{i}$, as recalled in Section III-A. The $\psi_{i}$ are then unambiguously computed as $\log \left(\Psi_{i}\right) / d$, since the distance $d$ between the antennas is smaller than half the wavelength of the signal, or equivalently $\left|\mathfrak{I}\left(\psi_{i} d\right)\right|<\pi$, where $\mathfrak{I}(\cdot)$ and $|\cdot|$ denote respectively the imaginary part and the absolute value of a complex number. Finally, the direction $\phi_{i}$ is found from the computed $\psi_{i}$ as $\phi_{i}=\arccos \left(-j \psi_{i} c / \omega\right)$. In general, the sparsity $n$, being the number of received signals, is also an unknown. The retrieval of $n$ is based on the singular value decomposition of some Hankel matrices, or density based cluster analysis, as discussed in Section III-B.

\section{A. Generalized eigenvalue approach}

With the samples $f_{m}$ we fill the Hankel matrices

$$
H_{n}^{(r)}:=\left[\begin{array}{cccc}
f_{r} & f_{r+1} & \ldots & f_{r+n-1} \\
f_{r+1} & f_{r+2} & \ldots & f_{r+n} \\
\vdots & \vdots & \ddots & \vdots \\
f_{r+n-1} & f_{r+n} & \ldots & f_{r+2 n-2}
\end{array}\right]
$$

where $r$ is an integer in the interval $[0, M-2 n+1]$. From the definition of these Hankel matrices and the samples $f_{m}$ we immediately find the factorization [6]

$$
H_{n}^{(r)}=V_{n} D_{\alpha} D_{\Psi}^{r} V_{n}^{T},
$$

where $V_{n}=\left(\Psi_{\ell}^{k-1}\right)_{k, \ell=1}^{n}$ is the Vandermonde matrix built with the distinct values $\Psi_{i}, i=1, \ldots, n$, and both matrices $D_{\alpha}=\operatorname{diag}\left(\alpha_{1}, \ldots, \alpha_{n}\right), D_{\Psi}=\operatorname{diag}\left(\Psi_{1}, \ldots, \Psi_{n}\right)$ are diagonal. Using the factorization (9) we find

$$
H_{n}^{(1)}-\lambda H_{n}^{(0)}=V_{n} D_{\alpha}\left(D_{\Psi}-\lambda I_{n}\right) V_{n}^{T} .
$$

Hence, the $\Psi_{i}$ are the generalized eigenvalues of the generalized eigenvalue problem

$$
H_{n}^{(1)} v_{i}=\lambda_{i} H_{n}^{(0)} v_{i}
$$

The coefficients $\alpha_{i}=S_{i}(t)$ are subsequently computed from the Vandermonde structured linear system

$$
\left[\begin{array}{ccc}
1 & \ldots & 1 \\
\Psi_{1} & \ldots & \Psi_{n} \\
\vdots & & \vdots \\
\Psi_{1}^{M-1} & \ldots & \Psi_{n}^{M-1}
\end{array}\right]\left[\begin{array}{c}
\alpha_{1} \\
\alpha_{2} \\
\vdots \\
\alpha_{n}
\end{array}\right]=\left[\begin{array}{c}
f_{0} \\
f_{1} \\
\vdots \\
f_{M-1}
\end{array}\right] .
$$

Note that in the noise-free case only $n$ of these $M$ equations are linearly independent. In the presence of noise, the linear system (11) is solved in the least squares sense for the $\alpha_{i}$. We can also solve the generalized eigenvalue problem (10) in a least squares sense [21], where the Hankel matrices defined by (8) are enlarged to dimension $(M-n) \times n$ to utilize all the available samples $f_{0}, \ldots, f_{M-1}$. In the sequel, we denote these rectangular Hankel matrices by $H_{n, M}^{(r)}$. Note that the Hankel matrices $H_{n, 2 n}^{(r)}$ are equal to the square Hankel matrices $H_{n}^{(r)}$.

\section{B. Determining the Sparsity $n$}

Until now we have assumed that the sparsity, or equivalently the number of received signals, $n$ is known. However, in practice this is not always the case. One possibility is to compute the numerical rank of Hankel matrices with increasing dimensions, since it is known that [26], [27, p. 603]

- $\operatorname{det} H_{N}^{(0)}=0$ only accidentally for $N<n$,

- $\operatorname{det} H_{n}^{(0)} \neq 0$,

- $\operatorname{det} H_{N}^{(0)}=0$ for $N>n$.

However, this method is not always reliable. In particular in the presence of noise, the method requires large values for $N$, meaning a large number of samples or antenna elements to estimate the sparsity $n$ correctly [28].

Another more reliable approach is based on the connection with Padé approximation theory [29]-[31]. Let us use the samples $f_{m}$ to construct a formal power series

$$
R(z)=\sum_{m=0}^{\infty} f_{m} z^{m}
$$

Using the definition of the samples $f_{m}$ we find

$$
\begin{aligned}
R(z)=\sum_{m=0}^{\infty}\left(\sum_{i=1}^{n} \alpha_{i} \Psi_{i}^{m}\right) z^{m} & \\
& =\sum_{i=1}^{n} \alpha_{i}\left(\sum_{m=0}^{\infty} \Psi_{i}^{m} z^{m}\right)=\sum_{i=1}^{n} \frac{\alpha_{i}}{1-\Psi_{i} z} .
\end{aligned}
$$

Hence, the formal power series $R(z)$ is that of a rational function of degree $n-1$ in the numerator and degree $n$ in the denominator. The consistency property of Padé approximation theory guarantees that in the noise-free case this rational function is recovered exactly by its $[n-1, n]_{R}$ Padé approximant of degree $n-1$ in the numerator and $n$ in the denominator for the formal power series $R(z)$ given by (12). But how to proceed in the noisy case?

Since $R(z)$ represents a rational function, the theorem of Nuttall-Pommerenke [32], [33] and the theory of Froissart doublets [34]-[38] may help us to determine the sparsity $n$ [28]. When adding a white Gaussian noise term $\epsilon_{m}$ to the output $f_{m}$ of the ULA, the power series $R(z)$ becomes

$$
R(z)+\epsilon(z)=\sum_{m=0}^{\infty}\left(f_{m}+\epsilon_{m}\right) z^{m} .
$$

The theorem of Nuttall-Pommerenke states that if the noisy power series $R(z)+\epsilon(z)$ is analytic throughout the complex plane except for a countable number of poles [32] and essential singularities [33], then the paradiagonal sequence of Padé approximants $\left\{[N-1, N]_{R+\epsilon}: N \in \mathbb{N}\right\}$ converges to $R(z)+$ $\epsilon(z)$ in measure on compact sets. This means that the measure of the set in the complex plane where the Padé approximants $[N-1, N]_{R+\epsilon}$ do not converge, goes to zero as $N$ tends to infinity. Note that this theorem does not say anything about pointwise or uniform convergence of the sequence, since the pointwise convergence is disrupted by $N-n$ unwanted polezero combinations of the Padé approximant $[N-1, N]_{R+\epsilon}$ : for every additional pole introduced by increasing $N$ beyond 
the sparsity $n$, there is a corresponding zero introduced in the numerator, almost canceling the pole locally. These pole-zero combinations are called Froissart doublets. Thanks to [37], [38], we know that the $n$ true poles are identified as the stable ones in successive Padé approximants $[N-1, N]_{R+\epsilon}$, while the $N-n$ spurious poles are randomly scattered for different Padé approximants or different realizations of the noise, hence they are distinguished by their instability [34], [35]. This means that these Froissart doublets offer a way to separate the received signals from the noise.

We therefore consider multiple snapshots to solve the problem [39]. Combining the results from multiple snapshots, the true poles (corresponding to the directions $\phi_{i}$ ) are identified as the stable poles, while the noise is represented by the randomly scattered ones. Hence, cluster analysis can be used to identify the stable poles as clusters and discard the remaining poles as noise. A density based method such as DBSCAN is a possible choice to correctly find the clusters [40]. DBSCAN requires two parameters $\mu$ and $\delta$. The first one is the minimal number of points $\mu$ required in the neighborhood of a point to be a core point and form a cluster. The second one is a distance parameter $\delta$ which defines the size of a neighborhood. The noise level influences the optimal choice for the parameters $\mu$ and $\delta$. Small values for the distance parameter $\delta$ allow us to detect dense clusters and thus very stable estimates for the correct $\Psi_{i}$, which is useful for low noise levels. In the case of high noise levels, a larger distance parameter $\delta$ is recommended, since this allows to detect wider clusters. When we choose $\mu$ equal to the number of snapshots, every pole has to be confirmed by all snapshots, resulting in an increased certainty that no incorrect angles $\phi_{i}$ are detected. On the other hand, when $\mu$ is smaller than the number of snapshots, it allows to discard erroneous results or noisy outliers. Also, in case of a low SNR, the number of snapshots can be increased while the value of $\mu$ can be relaxed. We even found that weak signals buried in noise, so with very low SNR compared to the other terms, can be detected [31] because of their structured character as opposed to the unstructured noise. In such a situation, multiple runs with different $\mu, \delta$ combinations can be considered.

So, to retrieve $n$ and the $\Psi_{i}, i=1, \ldots, n$ we solve the generalized eigenvalue problems

$$
H_{N, M}^{(1)} v_{i}=\lambda_{i} H_{N, M}^{(0)} v_{i}
$$

where $N$ is an integer in the interval $[n, M / 2]$, for every snapshot. Note that we have overestimated the sparsity $n$. Subsequently cluster analysis is used to retrieve the stable generalized eigenvalues and discard the remaining generalized eigenvalues since they represent the noise. Finally, the $\Psi_{i}$ are computed as the centers of mass of the retrieved clusters. It is recommended to take $N$, and in addition the number of antenna elements $M$, as large as possible. The new method is compared to standard ESPRIT on multiple snapshots in Section V.

In the next section we indicate how to break the Nyquist restriction $d<\lambda / 2$.

\section{Spatial Sub-NyQUist SAMPLing}

Let us now consider sparse antenna arrays where the distance $d$ between the antenna elements does not satisfy the spatial Nyquist condition $d<\lambda / 2$, where $\lambda$ is the wavelength of the received signal. Using a sparse array in contrast to a dense array does offer some advantages. Most importantly, for sparse arrays the mutual coupling effect is reduced, but also the angular resolution increases for the same number of antenna elements. Similar to the theory of co-prime arrays, we combine the results of two ULAs separately to retrieve the true angles from the aliased ones. However, our proposed method avoids the search step of matching and pairing the results obtained by the two separate sparse ULAs. Instead they are automatically linked by the new algorithm. Additionally, the proposed method also allows to validate the results and automatically retrieve the sparsity $n$, or the number of received signals [41]. This is similar to the clustering approach discussed in Section III-B.

\section{A. Sub-sampled Exponential Analysis}

When the spatial Nyquist bound is no longer satisfied, aliasing is introduced, which means that it is no longer possible to uniquely retrieve the $\psi_{i}$ from the $\Psi_{i}$, defined in Section III by (6), since it is no longer guaranteed that $\left|\mathfrak{I}\left(\psi_{i} d\right)\right|<\pi$. This aliasing problem is solved by using the output generated by a second ULA [17], as explained below.

We view both sparse ULAs as a sub-ULA of a virtual dense ULA, which does satisfy the spatial Nyquist bound. Note that we do not require the full dense ULA, only the antenna elements of the two sparse ULAs. A first ULA uses the output of the antennas $f_{m \sigma}$, where the spacing between the antenna elements is $\sigma d$. A second similar ULA uses the antenna elements shifted over a distance of $\rho d$ resulting in the output of the antenna elements $f_{m \sigma+\rho}$. The scale and shift parameters $\sigma \in \mathbb{N}_{0}$ and $\rho \in \mathbb{Z}_{0}$ are required to be co-prime, meaning $\operatorname{gcd}(\sigma, \rho)=1$, in order to fix the aliasing. In the following we denote the number of antennas in the first array by $M_{\sigma}$ and the number of antennas in the second array by $M_{\rho}$. Similar to the standard exponential analysis case, discussed in Section III, the number of antennas $M_{\sigma}$ and $M_{\rho}$ are restricted by the sparsity $n$. We require that $M_{\sigma} \geq 2 n$ and $M_{\rho} \geq n$. Note that the second sparse ULA need only consist of half the number of elements of the first sparse ULA. However, additional antenna elements can be used to solve the problem in a least squares sense. The set-up is illustrated in Fig. 2.

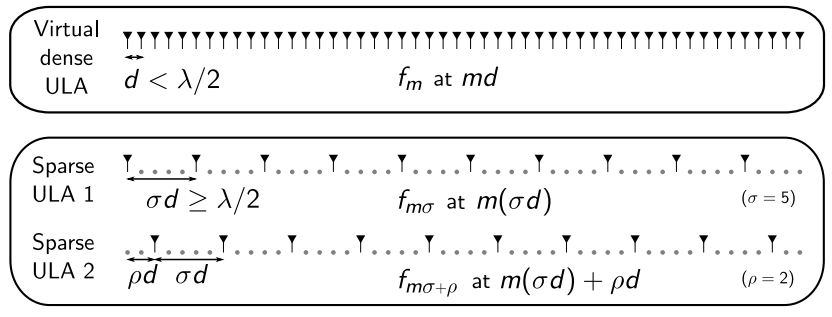

Fig. 2. Original dense array versus two sparse arrays. 
From the samples $f_{0}, f_{\sigma}, \ldots, f_{\left(M_{\sigma}-1\right) \sigma}$ we first compute the generalized eigenvalues of the model

$$
\begin{aligned}
f_{m \sigma}=\sum_{i=1}^{n} \alpha_{i} \Psi_{i}^{m \sigma}=\sum_{i=1}^{n} \alpha_{i}\left(\Psi_{i}^{\sigma}\right)^{m} & \\
m & =0, \ldots, M_{\sigma}-1,
\end{aligned}
$$

as described in Section III. Note that any one-dimensional implementation can be used, each with its own advantages and disadvantages, to retrieve the base terms $\Psi_{i}^{\sigma}$ associated with the samples taken at $0, \sigma d, \ldots,\left(M_{\sigma}-1\right) \sigma d$. From only the generalized eigenvalues $\Psi_{i}^{\sigma}$ it is not possible to retrieve the $\Psi_{i}$, since several possibilities exist, namely all the values in the set

$$
\left\{\exp \left(\psi_{i} d+\frac{2 \pi j}{\sigma} \ell\right): \ell=0, \ldots, \sigma-1\right\} .
$$

The second ULA is required to pinpoint the one correct value from this set. The samples collected by the second ULA satisfy

$$
\begin{aligned}
f_{m \sigma+\rho}=\sum_{i=1}^{n} \alpha_{i} \Psi_{i}^{m \sigma+\rho}=\sum_{i=1}^{n}\left(\alpha_{i} \Psi_{i}^{\rho}\right)\left(\Psi_{i}^{\sigma}\right)^{m}, \\
m=0, \ldots, M_{\rho}-1 .
\end{aligned}
$$

We find the same generalized eigenvalues $\Psi_{i}^{\sigma}$ but different coefficients compared to the model of the sparse ULA 1. Hence, both times a Vandermonde structured linear system with the same generators, but a different number of rows, is solved for the left-hand sides in (14) and in (16), to obtain both the values for the coefficients $\alpha_{i}$ in (14) and $\alpha_{i} \Psi_{i}^{\rho}$ in (16). Dividing the latter by the coefficients $\alpha_{i}$ results in the values for $\Psi_{i}^{\rho}$. Given the $\Psi_{i}^{\rho}$, again a set of possible values remains for each $\Psi_{i}$, namely

$$
\left\{\exp \left(\psi_{i} d+\frac{2 \pi j}{\rho} \ell\right): \ell=0, \ldots, \rho-1\right\} .
$$

Since we choose $\sigma$ and $\rho$ co-prime, the intersection of the sets (15) and (17) only contains the desired $\Psi_{i}$ [17]. This is illustrated in Fig. 3. Hence, the aliasing problem is

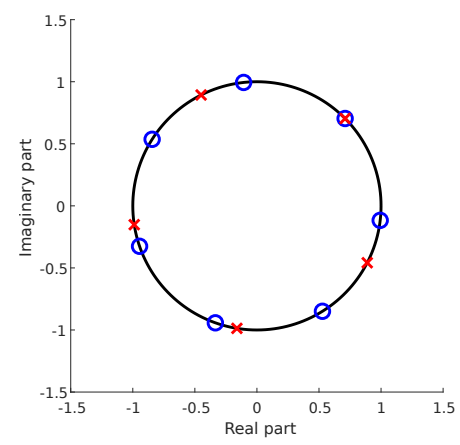

Fig. 3. Intersection of the sets (15) and (17) for $\sigma=7, \rho=5, \omega=1.5 \mathrm{GHz}$, $d=0.48 \lambda$ and the angle $\phi_{i}$ of the incoming signal being 105 degrees. No noise was added.

solved. Note that we already know which sets (15) and (17) correspond to each other due to the shared Vandermonde system, in contrary to the theory of co-prime arrays, where a search step is required to match the results of both ULAs. When noise is present, both sets do not intersect exactly. In this case we search for the two closest points in both sets. Note that while in theory $\sigma$ and $\rho$ may be chosen arbitrarily large, this is not the case in practice since noise can make it hard to point at the one correct value when a large number of points lie closer together in (15) and (17). Practically one would always design a system with as many sensors as possible. Both $\rho$ and $\sigma$ should be selected to be as small as possible for the desired angular resolution (since the resolution is ultimately proportional to $\sigma$ ), while the practical constraint on the total length must also be considered. Considering the noise effects, numerical experiments indicate that a smaller $\rho$ is more important than a smaller $\sigma$. Also the $\Psi_{i}$ values from (10) are usually less affected by noise than the $\Psi_{i}^{\rho}$ which are obtained as solution of a Vandermonde structured linear system.

The fact that the method solves (14) and (16) per snapshot and passes the individual results to a cluster detection algorithm, makes it computationally more efficient than traditional methods performing the analysis on much larger matrices containing the measurements of all snapshots simultaneously. In addition, the independence of all the smaller systems involved allows to parallelize the computation.

\section{B. Determining the Sparsity $n$}

In the sub-sampled case, we also use the connection with Padé approximation theory to determine the sparsity $n$. We first solve the generalized eigenvalue problem

$$
H_{N, M_{\sigma}}^{(1)} v_{i}=\lambda_{i} H_{N, M_{\sigma}}^{(0)} v_{i}, \quad n \leq N \leq\left\lfloor M_{\sigma} / 2\right\rfloor,
$$

where the Hankel matrices are filled with the output of the first sparse (not shifted) ULA, so the samples $f_{m \sigma}$. This yields $N$ generalized eigenvalues $\Psi_{i}^{\sigma}$, of which $n$ values correspond to the true angles and $N-n$ values correspond to the noise. Subsequently an $M_{\sigma} \times N$ and an $M_{\rho} \times N$ Vandermonde structured linear system is solved to retrieve $N$ estimates for the coefficients $\alpha_{i}$ and the $\Psi_{i}^{\rho}$. Once again, $n$ of these values correspond to the true angles, while the remaining $N-n$ model the noise. This process is repeated for all the snapshots. We collect all the results for multiple snapshots in two large sets: the set $A_{\sigma}$ for the first ULA and the set $A_{\rho}$ for the shifted ULA. Now cluster analysis identifies the $n$ true $\Psi_{i}^{\sigma}$ and $\Psi_{i}^{\rho}$ in the two sets $A_{\sigma}$ and $A_{\rho}$, as discussed in Section III-B.

Because of the shared Vandermonde structured linear system in both (14) and (16), the $\Psi_{i}^{\sigma}$ and $\Psi_{i}^{\rho}$ are matched automatically. Hence every element in the set $A_{\sigma}$ is directly connected with an element in the set $A_{\rho}$. By combining the DBSCAN results for $A_{\sigma}$ and $A_{\rho}$, ultimately three scenarios may occur [41]:

1) A cluster $C_{\sigma}$ is detected in the set $A_{\sigma}$ and its center of mass is used to estimate the generalized eigenvalue $\Psi_{i}^{\sigma}$. The points in the set $A_{\rho}$ connected to the elements in the cluster $C_{\sigma}$ also form a cluster $C_{\rho}$ in $A_{\rho}$ and their center of mass is used as estimate for the corresponding $\Psi_{i}^{\rho}$. From both centers of mass we recover the value for $\Psi_{i}$, as discussed in Section IV-A, and subsequently 
retrieve the angle $\phi_{i}$. This scenario is the standard one.

2) A cluster $C_{\sigma}$ is detected in the set $A_{\sigma}$. However, the points in the set $A_{\rho}$ connected to the cluster $C_{\sigma}$ do not form a cluster, hence the cluster $C_{\sigma}$ is discarded and not used in the computation for the values $\Psi_{i}$. Note that this effectively lowers the estimate for the sparsity $n$ since a cluster is removed.

3) A cluster $C_{\sigma}$ is detected in the set $A_{\sigma}$ and its center of mass is used to estimate the generalized eigenvalue $\Psi_{i}^{\sigma}$. The points in the set $A_{\rho}$ connected to the cluster $C_{\sigma}$ not all belong to a cluster $C_{\rho}$, since some of them are outliers. In this case the center of mass of only the clustered points is used as estimate for the corresponding $\Psi_{i}^{\rho}$. From both centers of mass we recover the value for $\Psi_{i}$, as discussed in Section IV-A, and subsequently retrieve the angle $\phi_{i}$.

Usually multiple DBSCAN runs are performed, starting with a high validation rate and relaxing it gradually (by decreasing $\mu$ and increasing $\delta$ ), until the clusters detected in $A_{\sigma}$ are not validated anymore by a cluster in $A_{\rho}$.

The computational effort required is thus solution of $K$ (number of snapshots) Hankel matrices of size $\left(M_{\sigma}-N\right) \times N$, and $2 K$ Vandermonde structured linear systems of size $M_{\rho} \times$ $N$. This process can be easily parallelized since all snapshots are uncoupled. In a last step, DBSCAN is performed on $K N$ different points to retrieve $n \leq N$ distinct clusters.

\section{EXAMPLES}

We illustrate the performance of our proposed method under the influence of noise and mutual coupling. In the first examples we only look at the effect of noise, hence no mutual coupling effects are considered. Subsequently, we consider some simulation examples which include full coupling under low noise levels, to isolate the effects of coupling only.

\section{A. Effects of noise}

First we demonstrate the behavior under influence of noise, mainly to illustrate the effect of the validation step. Therefore, we consider $n=10$ received signals, given in Table I. We use both standard ESPRIT and the new method with ESPRIT underlying to retrieve the 10 corresponding angles for increasing levels of noise. The additive noise is expressed in terms of SNR, which is defined by $20 \log _{10}\left(\|f\|_{2} /\|\epsilon\|_{2}\right)$, where $\|f\|_{2}$ and $\|\epsilon\|_{2}$ denote the 2-norm of respectively the sample and additive noise vector.
TABLE I

TABLE OF 10 RECEIVED SIGNALS.

\begin{tabular}{l|ccccc} 
& 1 & 2 & 3 & 4 & 5 \\
\hline Angle & 10 & 34 & 63 & 80 & 90 \\
Amplitude & 0.3 & 0.2 & 0.4 & 0.5 & 0.3 \\
Phase & $0.9 \pi$ & $1.2 \pi$ & $0.8 \pi$ & $0.7 \pi$ & $1.1 \pi$ \\
\hline \hline & 6 & 7 & 8 & 9 & 10 \\
\hline Angle & 96 & 124 & 141 & 154 & 166 \\
Amplitude & 0.4 & 0.7 & 0.2 & 0.5 & 0.4 \\
Phase & $0.7 \pi$ & $1.3 \pi$ & $1.2 \pi$ & $1.0 \pi$ & $1.1 \pi$
\end{tabular}

For the standard ESPRIT approach, a ULA of 60 antennas with a distance of $0.48 \lambda$ between the elements is considered. We also tell ESPRIT the correct number of signals, i.e. $n=10$. We solve the DOA problem using ESPRIT on 256 snapshots. In Fig. 4 at the top, the results of all 256 snapshots are shown together. We observe that ESPRIT works well for a high SNR, however for higher noise levels (around $10 \mathrm{~dB}$ ) the standard ESPRIT approach delivers unreliable results.

At the same time, our new approach (with ESPRIT as underlying method) also uses 60 antennas in total: a first sparse ULA of 30 antennas and a shifted ULA of 30 antennas, with a scale and shift parameter of respectively $\sigma=25$ and $\rho=14$. The distance between the virtual dense array elements is also chosen as $0.48 \lambda$ resulting in a total array size of more than $350 \lambda$. This might be an unrealistically large system for many applications, but the example serves to illustrate the efficacy of the proposed method even under such demanding conditions where both $\sigma$ and $\rho$ are large. As stated before, increasing $\sigma$ results in a more difficult root intersection (validation) problem. Since the method detects the number of signals $n$ automatically, $n$ need not be passed to the algorithm. For the clusters $C_{\sigma}$ we choose the DBSCAN parameters $\mu=0.8 \times 256=205$ with increasing equidistant $\delta$ values, namely $\delta=0.01,0.0825,0.155,0.2275,0.3$. For the $C_{\rho}$ clusters we take $\mu=0.6 \times 256=154$ with $\delta=0.5$, because they are usually less accurate, as already pointed out. At the bottom of Fig. 4 we find the results from 256 snapshots. For the lower noise levels, we clearly see that our method performs comparably. When the signals are perturbed with a lot of noise, we observe that the new method does not return all the angles, however, it also does not return unreliable results such as the stand-alone ESPRIT method. It detects that the signal is heavily perturbed, since the results are not validated by the cluster analysis and hence not all angles are recovered.

In a second experiment we compare our method to the coprime array method discussed in [42]. Although the array geometry for both methods is not the same, they are comparable in the sense that they consider two interleaved sparse arrays. In our case we have one array sub-sampled by a factor $\sigma$ and then shifted over $\rho$, while their method just combines the results of two sparse arrays, which are respectively sub-sampled by factors $\sigma_{1}$ and $\sigma_{2}$. Then a matching step is performed to be able to link the results of both sparse arrays. This matching is based on a projection of a two-dimensional point on a one- 

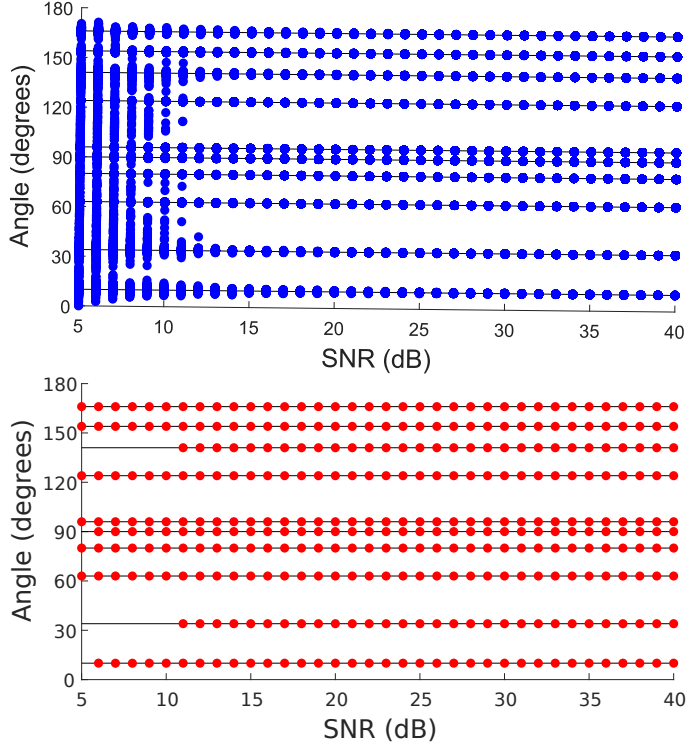

Fig. 4. The solution of the DOA problem given by Table I for increasing noise levels of both standard ESPRIT (top) and the new method (bottom).

dimensional line segment that corresponds to the entire angular domain due to the co-primeness of $\sigma_{1}$ and $\sigma_{2}$.

Similar to the first experiment, we use both methods to retrieve the $n=6$ angles given in Table II, for increasing noise levels. For this experiment we only consider 6 instead of 10 signals, since the matching in [42] becomes considerably worse for larger $n$-values.

TABLE II

TABLE OF 10 RECEIVED SIGNALS

\begin{tabular}{l|cccccc} 
& 1 & 2 & 3 & 4 & 5 & 6 \\
\hline Angle & 35.0 & 62.5 & 90 & 96.5 & 123.5 & 151 \\
Amplitude & 0.3 & 0.2 & 0.4 & 0.5 & 0.3 & 0.4 \\
Phase & $0.9 \pi$ & $1.2 \pi$ & $0.8 \pi$ & $0.7 \pi$ & $1.1 \pi$ & $0.7 \pi$
\end{tabular}

For both methods 256 snapshots are collected by two sparse ULAs of 20 elements each, where the distance between the elements of the virtual dense array is $0.48 \lambda, \sigma=\sigma_{1}=10$ and $\rho=\sigma_{2}=3$. We also pass the number of signals $n=6$ on to the co-prime array method, while our method is able to detect this number of impending signals automatically. For the cluster analysis we use DBSCAN on ULA1 with $\mu=218$ for $85 \%$ validation and $\delta=0.01,0.02,0.04,0.08,0.16,0.32$, while for ULA2 we require $70 \%$ validation with $\mu=179$ and $\delta=0.6$. The results are shown in Fig. 5, where for every noise level we plot the output of 100 different noise realizations to observe the effect of noise on both methods.

We see that for low noise levels, both methods perform comparably, however for higher noise levels the difference becomes clear. The co-prime arrays have trouble matching the results of both ULAs, hence, especially for high noise levels, we observe that mismatches are not uncommon and thus result in erroneously retrieved angles. On the other hand, our method may not validate all 6 angles in case of high noise levels, however it also does not yield any erroneous results. Note that it is possible to obtain all 6 angles for a low SNR with the proposed method when we are less strict on the validation part. However, this can lead to angles which are slightly less accurate than the ones returned in this experiment.
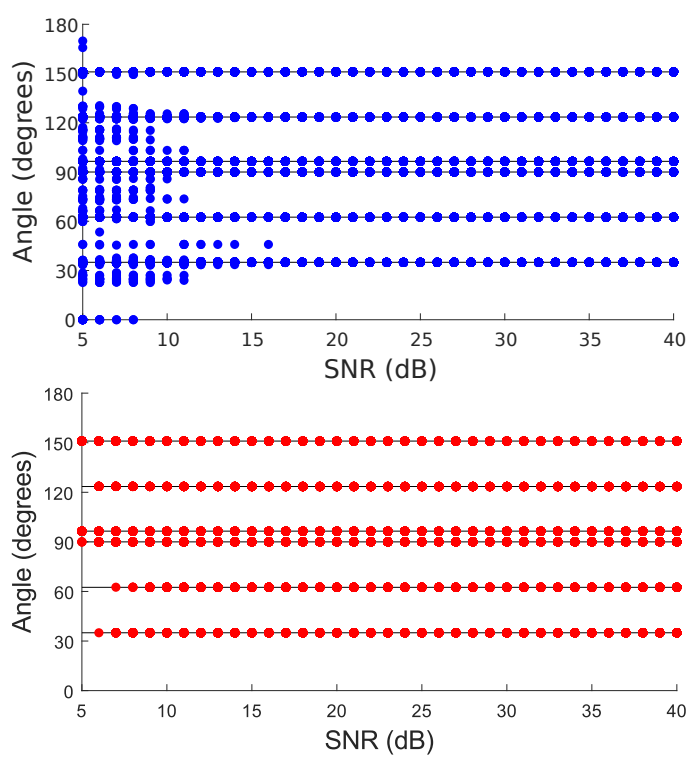

Fig. 5. The solution of the DOA problem given by Table II for increasing noise levels of both co-prime arrays (top) and the new proposed method (bottom).

Both ESPRIT and the sparse array method in [42] require solutions of matrices of the order $M \times K n$, which can be significantly slower than the series of smaller systems required for the new method.

\section{B. Effects of Mutual Coupling}

To illustrate the improved resolution of the algorithm over standard dense ULAs, as well as the effects of mutual coupling on the performance thereof, a 12 element dipole array is considered. The antennas have length $\lambda / 2$ and radius of $\lambda / 1000$ (thin wires), are oriented along the $z$-axis of a standard Cartesian coordinate system, and are arranged linearly along the $x$-axis. Spacing is defined as in Fig. 2, with $\sigma=11, \rho=5$, and $M_{\sigma}=M_{\rho}=6$, with the central feed points as reference positions. All elements are loaded with $50 \Omega$ sources, and fullwave method of moment (MoM) simulations using FEKO [43] are used to characterize the array system. For this experiment we fix SNR $=30 \mathrm{~dB}$, use 256 snapshots and investigate a range of values for $d \in[0.09 \lambda, 0.48 \lambda]$. Smaller values of $d$ correspond to denser arrays, while larger values result in sparse arrays with finer resolution and less mutual coupling.

The resolution of the system is evaluated by exciting the array with two monochromatic plane waves. The incoming directions of the $z$-polarized sources are both fixed at $\theta=90^{\circ}$, while the first is also fixed at $\phi_{1}=90^{\circ}$ and the second varied in $\phi_{2} \in\left[75^{\circ}, 90^{\circ}\right]$. Standard spherical coordinate system azimuth and polar angles are used for $\phi$ and $\theta$.

Three different spacing cases are investigated with $d=$ $0.09 \lambda, 0.3 \lambda, 0.48 \lambda$. For all cases 100 Monte-Carlo trial runs are performed, and the accuracy of the estimated angles is 
presented as the RMS value, over these trials, of the absolute difference between the estimated and the actual direction of arrival (for each of the signals). Since $n=2$ here, both the success rate as well as RMS accuracy of the DOA estimation algorithm is computed. The success rate is just the number of successful trials divided by the total number of trials as a percentage. If fewer than 2 angles are returned, the trial is counted as unsuccessful.

For comparison, results obtained by MUSIC on the same geometry are also included. To this end, the second signal is excited in quadrature with the first to remove all correlation between the signals. Results for the method presented here are identical to the uncorrelated case when correlated signals are used, while MUSIC fails in this case. To avoid any ambiguity issues for the sparse cases, the array manifold search space in the algorithm is restricted to the region $\left[\min \left(\phi_{2}\right)-\Delta \phi, \phi_{1}+\right.$ $\Delta \phi$, where the resolution $\Delta \phi$ is calculated using (1). Note that, for a standard $\lambda / 2$-spaced ULA with 12 elements, the resolution is expected to be around $10.5^{\circ}$, and resolutions for the other examples are indicated as vertical dotted lines on the result plots in Figs. 6 to 8 .

The results for the $d=0.09 \lambda$ case are shown in Fig. 6 . For this configuration $(\rho=5)$ the spacing results in a $0.45 \lambda$ separation between the closest elements. The worst case mutual coupling is around $-15.3 \mathrm{~dB}$, very similar to the value obtained from a classical $\lambda / 2$-spaced ULA. Since the maximum separation here is only $0.54 \lambda$, standard dense array techniques can be used near broadside without the risk of ambiguity. Clearly, the new method suggested in this
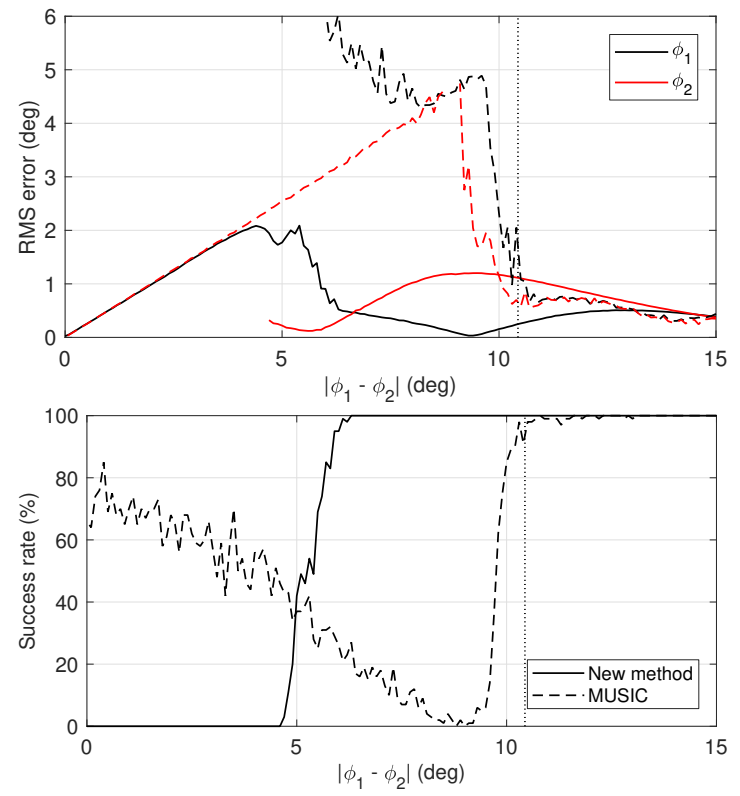

Fig. 6. Performance of the presented method (solid line) and MUSIC (dashed line) for a $d=0.09 \lambda$ spaced array system. The top panel shows the accuracy of the extracted angles of arrival, and the bottom panel the success rates of the methods. The vertical line indicates the expected minimum Rayleigh resolution limit.

work, performs significantly better than MUSIC in terms of resolution. Both incoming signals can be resolved reliably from about half the Rayleigh limit.
Results for $d=0.3 \lambda$ and $d=0.48 \lambda$ are shown in Figs. 7 and 8 respectively. Similar results as before are obtained,
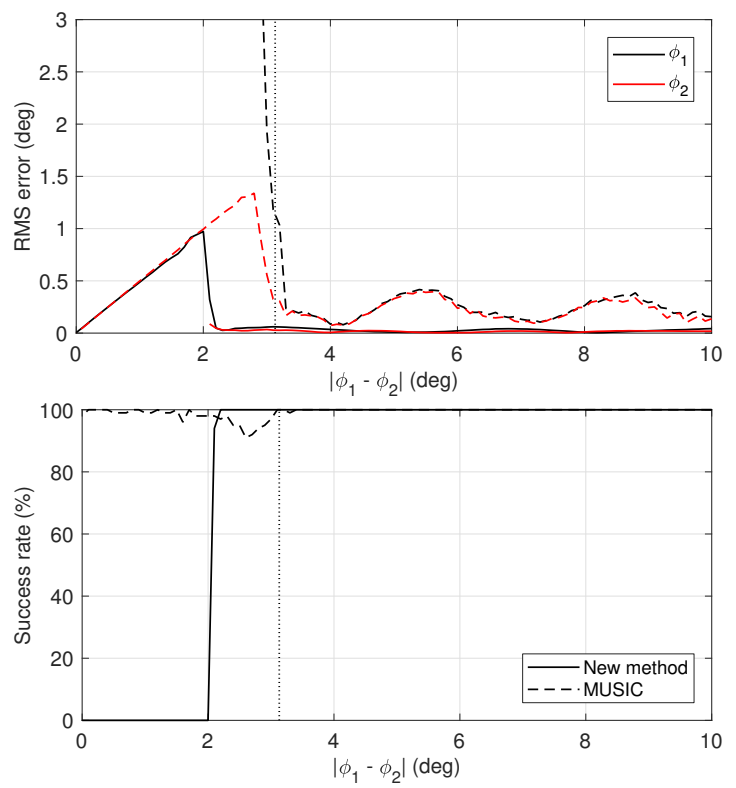

Fig. 7. Performance of the presented method (solid line) and MUSIC (dashed line) for a $d=0.3 \lambda$ spaced array system. Top and bottom panel as in 6 . The vertical line indicates the expected minimum Rayleigh resolution limit.
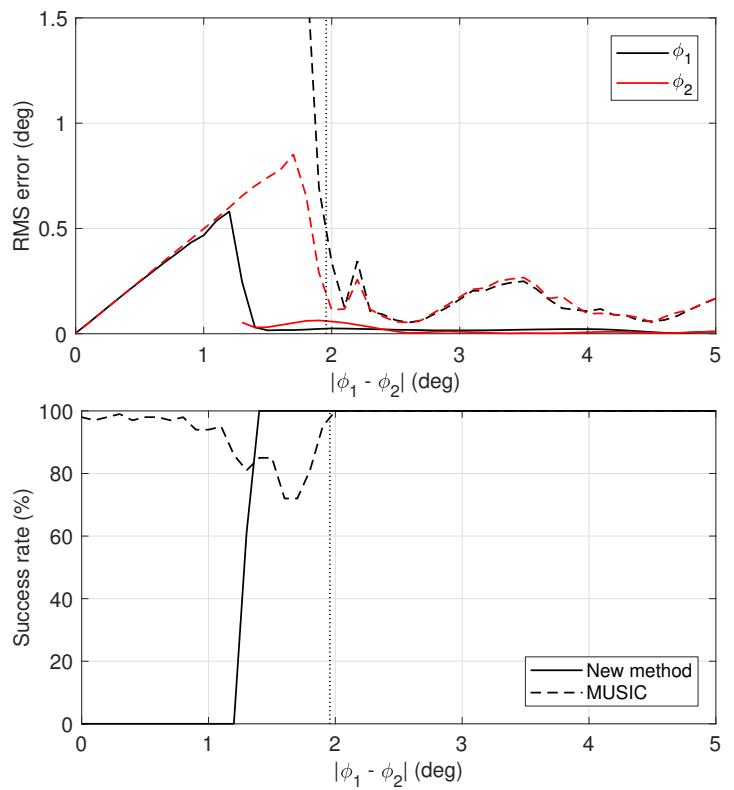

Fig. 8. Performance of the presented method (solid line) and MUSIC (dashed line) for a $d=0.48 \lambda$ spaced array system. Top and bottom panel as in 6 .

where the present method not only displays better resolution than MUSIC, but also lower error values. It must be stressed here that MUSIC cannot really be used in these cases, since ambiguous results are found when searching over the full array manifold (as is normally done). The ambiguity is artificially removed through prior knowledge of the incoming signal directions for MUSIC, while the present method returns unique results. Compared to the narrower spaced results, increasing $d$ results in reduced mutual coupling and thus improved accuracy 
in angle estimation for both methods. Note again that the resolution for the $d=0.48 \lambda$ case is about a factor 5 better than that achievable with a standard ULA, and this value can be further increased by increasing the scale and shift parameters $\sigma$ and $\rho$ in the array.

\section{CONCLUSION}

A new method is presented for DOA estimation in sparse regularly spaced array systems. The method can be used on top of any exponential analysis method and completely removes the dense Nyquist spacing requirement. Instead, it only requires that the elements are arranged in two uniform arrays with the one an end-fire translated version of the other. By enforcing a co-prime relation between the element spacing and the translation distance, aliasing due to the spacial sparsity of the elements is removed. The larger spacing allows improved resolution for a fixed number of sensors and reduced mutual coupling between the antenna elements. Furthermore, the method provides an accurate estimate of the number of signals impinging on the system without any prior knowledge, while there is no requirement that the respective signals be uncorrelated. Distinct, fully correlated signals, such as those that occur in a multi-path environment, can thus accurately be distinguished using this new method. Several examples are presented investigating both the performance in increasing noise, as well as increasing mutual coupling scenarios. In all of these, the new method performs better than standard techniques on a variety of metrics. Work is currently ongoing towards expansion of the method to the planar case, which will be reported in a subsequent paper. A prototype implementation is also under development.

\section{ACKNOWLEDGMENT}

This work is based on the research supported in part by the National Research Foundation of South Africa (Grant Numbers: 75322 and 105717).

\section{REFERENCES}

[1] Z. Chen, G. Gokeda, and Y. Yu, Introduction to Direction-of-Arrival Estimation. Norwood, MA, USA: Artech House, 2010.

[2] A. P. Chippendale and G. Hellbourg, "Interference mitigation with a modified ASKAP phased array feed on the $64 \mathrm{~m}$ Parkes radio telescope," in Int. Conf. Electromag. Advanced Applications (ICEAA), Verona, Italy, Sept 2017, pp. 948-951.

[3] F. Boccardi, R. W. Heath, A. Lozano, T. L. Marzetta, and P. Popovski, "Five disruptive technology directions for 5G," IEEE Commun. Mag., vol. 52, no. 2, pp. 74-80, Feb. 2014.

[4] R. Schmidt, "Multiple emitter location and signal parameter estimation," IEEE Trans. Antennas Propag., vol. 34, no. 3, pp. 276-280, 1986.

[5] R. Roy and T. Kailath, "ESPRIT-estimation of signal parameters via rotational invariance techniques," IEEE Trans. Acoust. Speech Signal Process., vol. 37, no. 7, pp. 984-995, Jul. 1989.

[6] Y. Hua and T. K. Sarkar, "Matrix pencil method for estimating parameters of exponentially damped/undamped sinusoids in noise," IEEE Trans. Acoust. Speech Signal Process., vol. 38, pp. 814-824, 1990.

[7] A. Moffet, "Minimum-redundancy linear arrays," IEEE Trans. Antennas Propag., vol. 16, no. 2, pp. 172-175, Mar 1968.

[8] P. Pal and P. P. Vaidyanathan, "Nested arrays: A novel approach to array processing with enhanced degrees of freedom," IEEE Trans. Signal Process., vol. 58, no. 8, pp. 4167-4181, Aug 2010.

[9] P. P. Vaidyanathan and P. Pal, "Sparse sensing with co-prime samplers and arrays," IEEE Transactions on Signal Processing, vol. 59, no. 2, pp. 573-586, Feb 2011.
[10] A. Paulraj and T. Kailath, "Direction of arrival estimation by eigenstructure methods with unknown sensor gain and phase," in IEEE Int. Conf. Acoustics, Speech, and Signal Process., vol. 10, Apr 1985, pp. 640-643.

[11] B. Friedlander and A. J. Weiss, "Direction finding in the presence of mutual coupling," IEEE Trans. Antennas Propag., vol. 39, no. 3, pp. 273-284, Mar 1991.

[12] F. Sellone and A. Serra, "A novel online mutual coupling compensation algorithm for uniform and linear arrays," IEEE Trans. Signal Process., vol. 55, no. 2, pp. 560-573, Feb 2007.

[13] P. Rocca, M. A. Hannan, M. Salucci, and A. Massa, "Single-snapshot DoA estimation in array antennas with mutual coupling through a multiscaling BCS strategy," IEEE Trans. Antennas Propag., vol. 65, no. 6, pp. 3203-3213, June 2017.

[14] Z. Ye, J. Dai, X. Xu, and X. Wu, "DOA estimation for uniform linear array with mutual coupling," IEEE Trans. Aerosp. Electron. Syst., vol. 45, no. 1, pp. 280-288, Jan 2009.

[15] Y. Wang, M. Trinkle, and B. W.-H. Ng, "DOA estimation under unknown mutual coupling and multipath with improved effective array aperture," Sensors, vol. 15, no. 12, pp. 30856-30869, 2015.

[16] E. BouDaher, F. Ahmad, M. G. Amin, and A. Hoorfar, "Effect of mutual coupling on direction-of-arrival estimation using sparse dipole arrays," in IEEE Int. Symp. Antennas Propag., Fajardo, Puerto Rico, 2016.

[17] A. Cuyt and W.-s. Lee, "How to get high resolution results from sparse and coarsely sampled data," Appl. Comput. Harmon. Anal., 2018, to appear.

[18] Z. Tan, Y. C. Eldar, and A. Nehorai, "Direction of arrival estimation using co-prime arrays: A super resolution viewpoint," IEEE Trans. Signal Process., vol. 62, no. 21, pp. 5565-5576, Nov 2014.

[19] P. Pal and P. P. Vaidyanathan, "Coprime sampling and the MUSIC algorithm," in 2011 Digital Signal Processing and Signal Processing Education Meeting (DSP/SPE), Jan 2011, pp. 289-294.

[20] G. Golub, P. Milanfar, and J. Varah, "A stable numerical method for inverting shape from moments," SIAM J. Sci. Comput., vol. 21, pp. 1222 1243, 1999.

[21] S. Das and A. Neumaier, "Solving overdetermined eigenvalue problems," SIAM Journal on Scientific Computing, vol. 35, no. 2, pp. A541-A560, 2013.

[22] G. Beylkin and L. Monzón, "On approximation of functions by exponential sums," Appl. Comput. Harmon. Anal., vol. 19, no. 1, pp. 17-48, 2005.

[23] D. Potts and M. Tasche, "Parameter estimation for exponential sums by approximate Prony method," Signal Process., vol. 90, pp. 1631-1642, 2010.

[24] — "Parameter estimation for nonincreasing exponential sums by Prony-like methods," Linear Algebra Appl., vol. 439, no. 4, pp. 10241039, 2013.

[25] R. de Prony, "Essai expérimental et analytique sur les lois de la dilatabilité des fluides élastiques et sur celles de la force expansive de la vapeur de l'eau et de la vapeur de l'alkool, à différentes températures," J. Ec. Poly., vol. 1, pp. 24-76, 1795.

[26] E. Kaltofen and W.-s. Lee, "Early termination in sparse interpolation algorithms," J. Symbolic Comput., vol. 36, no. 3-4, pp. 365-400, 2003, International Symposium on Symbolic and Algebraic Computation (ISSAC'2002) (Lille).

[27] P. Henrici, Applied and computational complex analysis I. New York: John Wiley \& Sons, 1974.

[28] A. Cuyt, M. Tsai, M. Verhoye, and W.-s. Lee, "Faint and clustered components in exponential analysis," Appl. Math. Comput., vol. 327, pp. 93-103, 2018

[29] L. Weiss and R. McDonough, "Prony's method, $Z$-transforms, and Padé approximation," SIAM Rev., vol. 5, pp. 145-149, 1963.

[30] Z. Bajzer, A. C. Myers, S. S. Sedarous, and F. G. Prendergast, "PadéLaplace method for analysis of fluorescence intensity decay," Biophys J., vol. 56, no. 1, pp. 79-93, 1989.

[31] A. Cuyt and W.-s. Lee, "Sparse interpolation and rational approximation," ser. Contemporary Mathematics, D. Hardin, D. Lubinsky, and B. Simanek, Eds., vol. 661. Providence, RI: American Mathematical Society, 2016, pp. 229-242.

[32] J. Nuttall, "The convergence of Padé approximants of meromorphic functions," J. Math. Anal. Appl., vol. 31, pp. 147-153, 1970.

[33] C. Pommerenke, "Padé approximants and convergence in capacity," $J$. Math. Anal. Appl., vol. 41, pp. 775-780, 1973.

[34] P. Barone, "On the distribution of poles of Pad approximants to the Z-transform of complex Gaussian white noise," J. Approx. Theory, vol. 132, no. 2, pp. $224-240,2005$. 
[35] L. Perotti, D. Vrinceanu, and D. Bessis, "Enhanced frequency resolution in data analysis," American J. Comput. Math., vol. 3, no. 3, pp. 242-251, 2013.

[36] D. Bessis, "Padé approximations in noise filtering," J. Comput. Appl. Math., vol. 66, pp. 85-88, 1996.

[37] J. Gilewicz and M. Pindor, "Padé approximants and noise: a case of geometric series," J. Comput. Appl. Math., vol. 87, pp. 199-214, 1997.

[38] — - "Padé approximants and noise: rational functions," J. Comput. Appl. Math., vol. 105, pp. 285-297, 1999.

[39] M. Briani, A. Cuyt, and W. Lee, "VEXPA: Validated EXPonential Analysis through regular subsampling," Universiteit Antwerpen, ArXiv e-print 1709.04281 [math.NA], 2017.

[40] M. Ester, H.-P. Kriegel, J. Sander, and X. Xu, "A density-based algorithm for discovering clusters in large spatial databases with noise," in Proceedings of the Second International Conference on Knowledge Discovery and Data Mining, ser. KDD'96. Portland, Oregon: AAAI Press, 1996, pp. 226-231.

[41] M. Briani, A. Cuyt, and W. Lee, "Validated exponential analysis for harmonic sounds," in DAFX17, 20th international conference on digital audio effects, 2017.

[42] Z. Weng and P. M. Djurić, "A search-free DOA estimation algorithm for coprime arrays," Digital Signal Processing, vol. 24, pp. 27 - 33, 2014.

[43] Altair Development S.A. (Pty) Ltd, Stellenbosch, South Africa. FEKO, Suite 2017. [Online]. Available: https://altairhyperworks.com/feko/

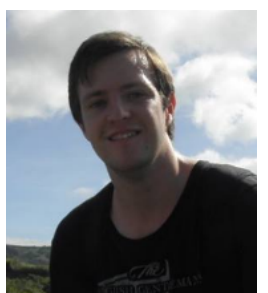

Ferre Knaepkens is a $\mathrm{PhD}$ student at the Department of Mathematics and Computer Science of the University of Antwerp. In 2017 he obtained, with greatest distinction, the degree Master of Science in Mathematics from the same university. In view of his strong interest in numerical and applied mathematics, he subsequently started working on a research project with the Computational Mathematics (CMA) group of the University of Antwerp.

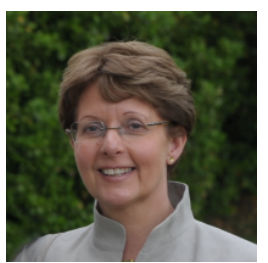

Annie Cuyt is full professor at the Department of Mathematics and Computer Science of the University of Antwerp and is a lifetime member of the Flemish Royal Academy of Belgium for Science and the Arts since 2014. She received her Doctor Scientiae degree in 1982 from the same university, summa cum laude and with the felicitations of the jury. Subsequently she was a Research fellow with the Alexander von Humboldt Foundation (Germany) and she was honoured with a Masuda Research Grant (Japan). She is the author of more than 200 peerreviewed publications in international journals and conference proceedings, the author or editor of several books and the organizer of a number of international events. Her current interests are in numerical approximation theory and its numerous applications in scientific computing. The latter vary from bioinformatics over different engineering applications (telecommunication, radar imaging, EM modeling, exponential analysis, ...) to computer graphics. In view of her expertise she served on many national and international science foundation boards.

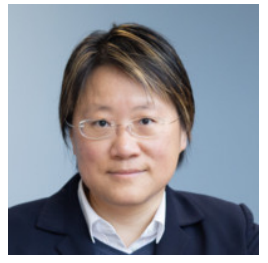

Wen-shin Lee (M'11) is a lecturer at the Division of Computing Science and Mathematics of the University of Stirling in the UK. She obtained her $\mathrm{PhD}$ degree in Computational Mathematics in 2001 from North Carolina State University in the US. Afterwards, she was a postdoctoral fellow at the University of Waterloo, Canada and at INRIA Sophia Antipolis, France. In 2005, she moved to Belgium and became a member of the Computational Mathematics research group at the University of Antwerp. Her research background is from computer algebra and symbolic-numeric computation. Her current focus is on exponential analysis, especially its connection to sparse interpolation and applications in signal processing. She is the editor of ACM Communications in Computer Algebra.

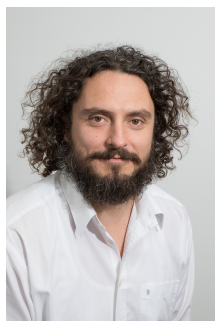

Dirk de Villiers (S'05-M'08-SM'15) received the B.Eng and Ph.D. degrees in electrical and electronic engineering from the University of Stellenbosch, Stellenbosch, South Africa in 2004 and 2007 respectively.

From 2008 to 2009 he was a post-doctoral fellow at the University of Stellenbosch working on antenna feeds for the South African SKA program. He is currently a Professor at Stellenbosch University, where he holds the SARChI Research Chair in Antenna Systems for SKA. He has spent several months as visiting researcher at Antwerp University in Antwerp, Belgium and at Chalmers University of Technology in Gothenburg, Sweden. Since 2010 he has regularly worked on contract for EMSS Antennas (Pty) Ltd in Stellenbosch, South Africa, on the design of the reflector antenna systems for the MeerKAT, SKA, and ngVLA radio telescopes. His main research interests include reflector antennas for radio astronomy, as well as surrogatebased optimization of microwave components.

He served as chair of the South African IEEE joint AP/MTT/EMC chapter between 2017-2018, and is a senior member of URSI. 